\title{
Ocular Chemical Burn with an Unusual Presentation: A Case Report
}

\author{
Malihe Nikandish (iD) ${ }^{1, *}$ \\ ${ }^{1}$ Ophthalmology Department, Valiasr Hospital, Birjand University of Medical sciences, Birjand, Iran \\ "Corresponding author: Ophthalmology Department, Valiasr Hospital, Birjand University of Medical sciences, Birjand, Iran. Email: nikandishm@bums.ac.ir \\ Received 2021 January 24; Revised 2021 January 30; Accepted 2021 January 31.
}

\begin{abstract}
Introduction: Ocular chemical injury is an ophthalmic emergency that may be challenging to manage. Here, we presented a case of persistent corneal astigmatism secondary to chemical burn with superficial limbal injury and no corneal involvement.

Case Presentation: The case was a 36-year-old man who presented with a chemical acid injury in the right eye. He had sectorial superficial involvement of inferior limbus from 4 to 5 o'clock hours, and the cornea was clear. Corneal topography showed high irregular astigmatism that was not corrected with glass. In long-term follow-up, changes in topographic parameters happened very slowly.

Conclusions: In conclusion, optical corneal changes should be considered in the ocular surface chemical burn, especially in sectorial involvement despite clear cornea.
\end{abstract}

Keywords: Eye, Chemical Burn, Astigmatism

\section{Introduction}

Ocular chemical injury is an ophthalmic emergency that its prognosis depends on the type of chemical substance, the amount of involvement, and prompt handling (1). Ocular chemical burn management can be challenging, particularly in cases with severe burns. Ocular surface tissue, including the eyelid, the conjunctiva, and the cornea, may show serious and irreversible damage in severe chemical injuries (2).

Classification of severity of chemical burn contains both limbal involvements in clock hours and the percentage of conjunctival involvement. Limbal involvement can be superficial and transient or deeper and permanent. Permanent limbal involvement is usually associated with loss of limbal stem cells, which naturally promotes regeneration of normal corneal epithelium. In superficial involvements, surface staining with deeper stem cells surviving may be found (3). Such problems may be improved within few days without a significant sequel.

In this study, we presented a case of high long-term persistent corneal astigmatism secondary to chemical burn with superficial limbal involvement and no corneal involvement.

\section{Case Presentation}

Our case was a 36-year-old man who presented with a chemical acid injury in the right eye. The study protocol and examinations were reviewed and approved by the Ethics Committee of the Birjand University of Medical Sciences. Besides, written informed consent was obtained.

Standard medical therapy was provided, including irrigation of the involved eye by sterile saline solution, administration of topical chloramphenicol, preservative-free tear substitutes, topical betamethasone and homatropine, and orally administered vitamin C. His uncorrected visual acuity (UCVA) was 2/10 and 10/10 in the right and left eye, respectively. External examination showed red-eye with ciliary injection. Eyelid skin was uninvolved. Conjunctival involvement started from the lower bulbar conjunctiva up to the inferior limbus around one clock hour in the right eye (Figure 1A). Slit-lamp examination revealed no corneal epithelial defect and opacity. Positive fluorescein uptake was noted in the above-mentioned areas of conjunctival involvement. Intraocular pressures were normal in both eyes. Diagnosis of chemical injury, right eye grade $2(<30 \%$ conjunctival involvement, as per Dua's classification), was confirmed (3).

After one week of medical treatment, conjunctival fluorescein staining was improved, but the patient complains

Copyright (c) 2021, Modern Care Journal. This is an open-access article distributed under the terms of the Creative Commons Attribution-NonCommercial 4.0 International License (http://creativecommons.org/licenses/by-nc/4.0/) which permits copy and redistribute the material just in noncommercial usages, provided the original work is properly cited. 

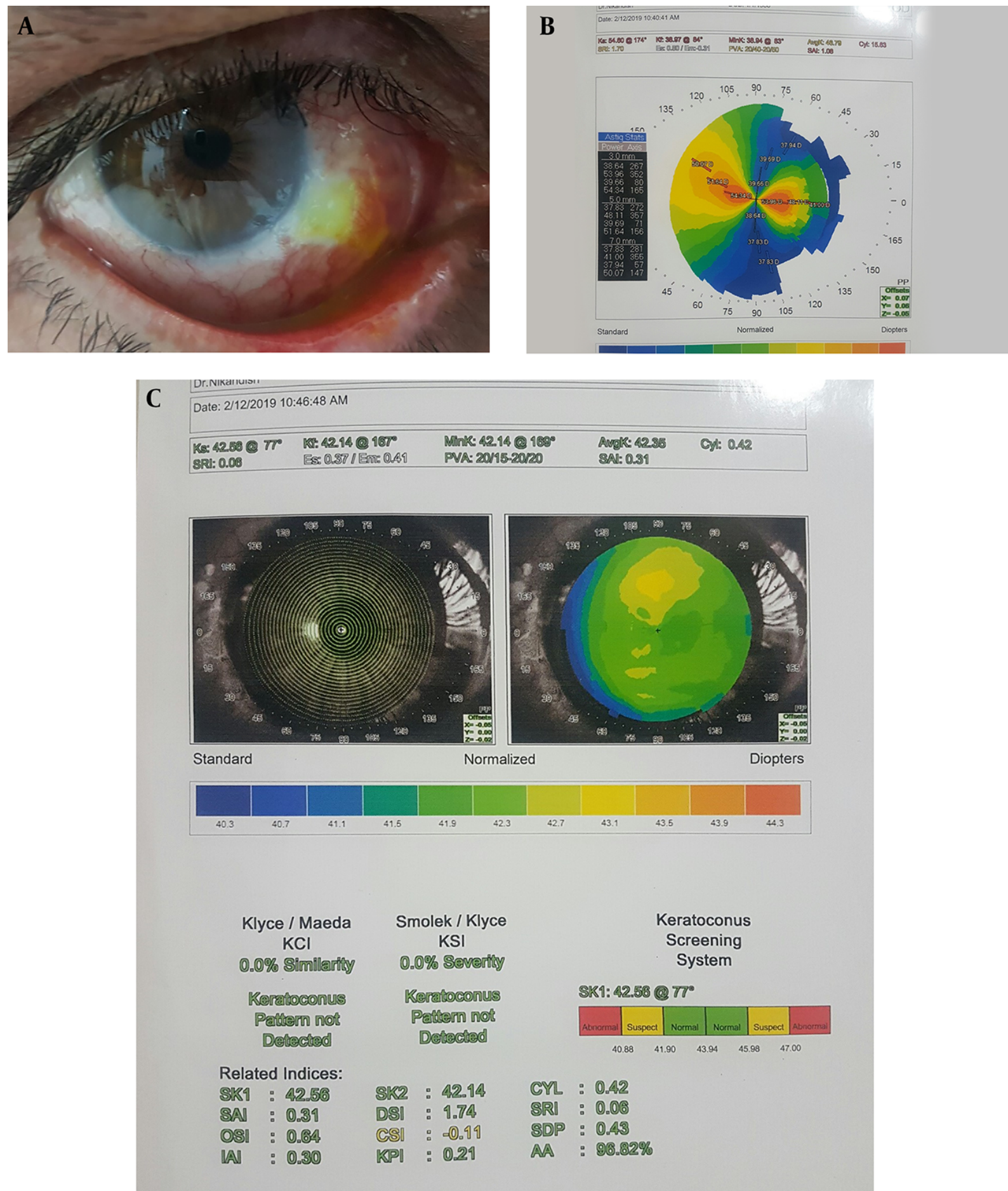

Figure 1. A) Conjunctival and limbal involvement in the right eye. B) Corneal topography of the right eye in acute phase showed high astigmatism (15D). C) Normal corneal topography of the left eye. 
of decreased visual acuity. Refractive error of right eye shows high astigmatism, and despite clear cornea and no visual axis involvement, corrected visual acuity was 6/10. The other eye was normal and visual acuity was 10/10. Thus, for further evaluation, corneal topography was performed on both eyes, which interestingly showed high against the rule astigmatism in the right eye with excessive flattening in the meridian of limbal involvement and normal topography in the left eye (Figures $1 \mathrm{~B}$ and $1 \mathrm{C}$ ). Except for ocular surface, other eye examinations, including funduscopy, were normal. Since the patient had a driving license with no history of vision loss and the topography of the other eye was normal (Figure 1C), and the flat axis in the topography coincided with the location of the lesion in the limbus, the topographic changes were attributed to the lesion.

In the follow-up examination, two months later, topographic findings were persistent, but the amount of corneal astigmatism decreased (Figure 2A). However, the patient did not come back for as long as several months. In the last examination, after 22 months, BCVA in the right eye was $8 / 10$, and corneal astigmatism was decreased but was not completely improved in the last topography (Figure $2 \mathrm{~B})$.

\section{Discussion}

In the present study, we described an unusual longterm complication of ocular surface chemical burn. This complication may be easily missed, which will result in Sight-threatening effects, especially in children. In general, at the acute stage of an ocular chemical burn, special attention should be to promoting epithelialization, optimizing tear film, and decreasing inflammation of the ocular surface. An overwhelming long-term sequel of severe chemical eye injury is named limbal stem cell deficiency (LSCD), which presents as corneal conjunctivalisation, fornical shortening, symblepharon formation, and ciccatricial entropion or ectropion (4). The advancement of treatment approaches, predominantly limbal stem cell transplantation, has resulted in improved visual and surface outcomes in severe chronic phases of the disease (5).

In our case, severe corneal astigmatism, despite the absence of corneal involvement, may be due to collagen shrinkage of the sclera that induces flattening effect of the nearby cornea and steepens the effects of meridians perpendicular. Based on our experience in this patient, collagen remodeling required a long time to be restored. Therefore, in sectorial limbal involvement, even if it was superficial and heals quickly, special attention should be paid to optical aspects of the eye. Recovery time may be long, and visual optical rehabilitation should be considered during this period, especially in children. This should be considered in parallel with other measures such as optimizing tear film and ocular surface reconstruction, and limbal stem cell remodeling. To the best of our knowledge, this is the first case of severe persistent corneal astigmatism secondary to chemical burn without obvious corneal involvement.

In conclusion, in sectoral limbal involvement in chemical injury, even in superficial cases, corneal optical parameters can be extremely changed despite the clear cornea. Therefore, awareness of ophthalmologists and other health care providers about this occult complication and its proper handling would be useful for preventing visual deprivation, especially for children of amblyopic ages.

\section{Footnotes}

Authors' Contribution: All parts of the manuscript(MN). Conflict of Interests: None.

Ethical Approval: The study protocol and examinations were reviewed and approved by the Ethics Committee of Birjand University of Medical Sciences (IR.BUMS.REC.1399.481).

Funding/Support: None.

Informed Consent: Written informed consent was obtained from the patient.

\section{References}

1. Singh S, Narang P, Mittal V. Oral mucosal grafting combined with tenonplasty for ocular surface and lid margin reconstruction in an atypical sectorial chemical burn. BMJ Case Rep. 2017;2017. doi: 10.1136/bcr-2017-221107. [PubMed: 28835428]. [PubMed Central: PMC5624005].

2. Liu T, Zhai H, Xu Y, Dong Y, Sun Y, Zang X, et al. Amniotic membrane traps and induces apoptosis of inflammatory cells in ocular surface chemical burn. Mol Vis. 2012;18:2137-46. [PubMed: 22876141]. [PubMed Central: PMC3413422].

3. Dua HS, King AJ, Joseph A. A new classification of ocular surface burns. Br J Ophthalmol. 2001;85(11):1379-83. doi: 10.1136/bjo.85.11.1379. [PubMed: 11673310]. [PubMed Central: PMC1723789].

4. Haring RS, Canner JK, Schneider EB. Chemical ocular burn epidemiology-dealing with missing values-reply. JAMA Ophthalmol. 2017;135(8):893. doi: 10.1001/jamaophthalmol.2017.1849. [PubMed: 28654975].

5. Bizrah M, Yusuf A, Ahmad S. An update on chemical eye burns. Eye (Lond). 2019;33(9):1362-77. doi: 10.1038/s41433-019-0456-5. [PubMed: 31086244]. [PubMed Central: PMC7002428]. 

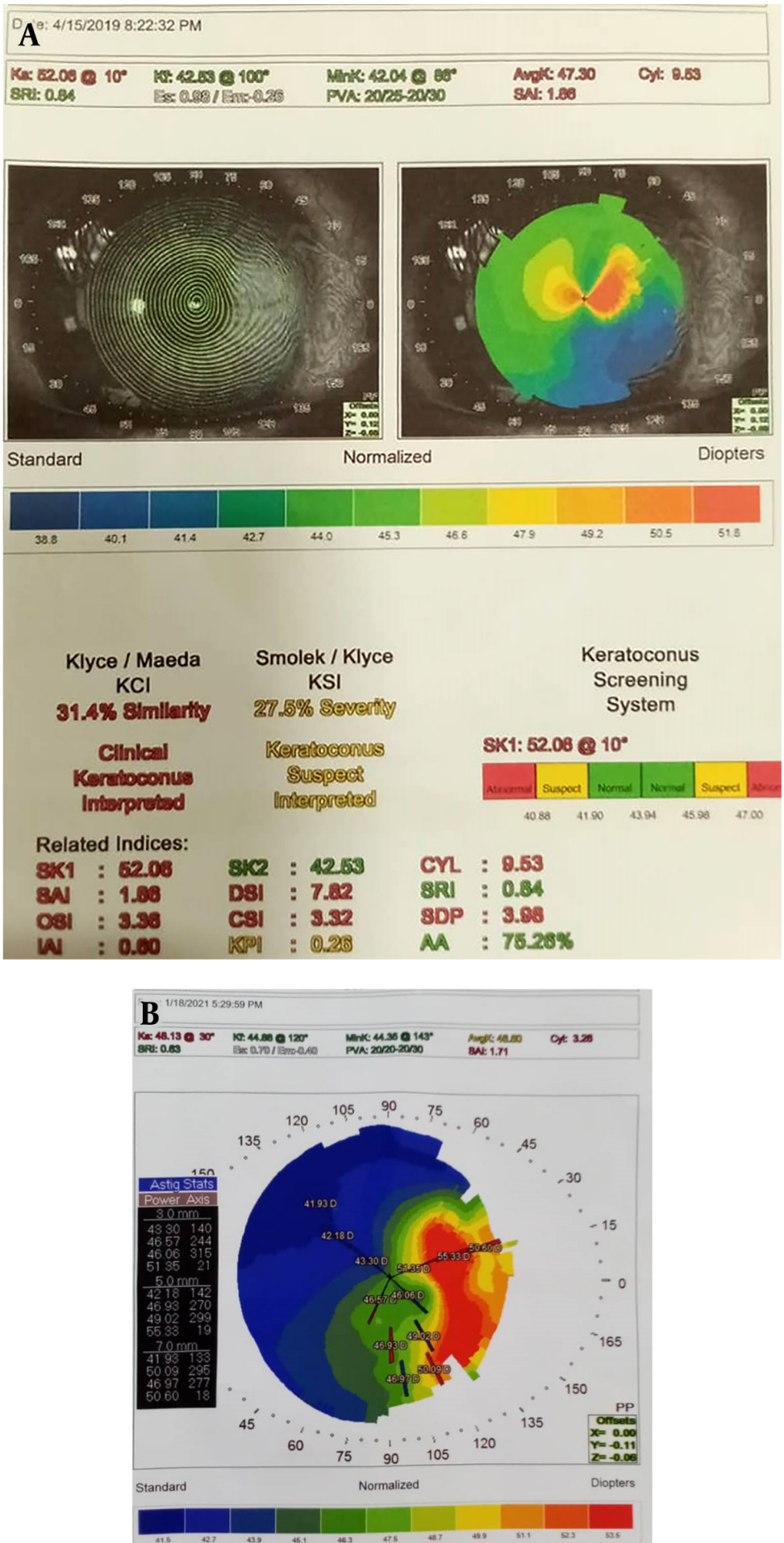

Figure 2. A) Corneal topography of the right eye two month later showed partially improvement of astigmatism (9D). B) Corneal topography of the right eye 22 months later showed even residual astigmatism (3D). 